\title{
A importância de um plano de contingência na farmácia hospitalar frente a
}

\section{pandemia pelo novo coronavírus}

The importance of a contingency plan in the hospital pharmacy against the pandemic by the new coronavirus

\section{La importancia de un plan de contingencia en la farmacia del hospital contra la pandemia por el}

\author{
nuevo coronavirus
}

Recebido: 11/06/2021 | Revisado: 19/06/2021 | Aceito: 24/06/2021 | Publicado: 26/06/2021

\author{
Cynthia Antunes do Nascimento Costa \\ ORCID: https://orcid.org/0000-0001-9427-1538 \\ Universidade Estácio de Sá, Brasil \\ E-mail: cynthia.antunescosta@gmail.com \\ Dayse dos Santos Bastos da Costa \\ ORCID: https://orcid.org/0000-0002-0975-8403 \\ Universidade Estácio de Sá, Brasil \\ E-mail: dayse.bastos@yduqs.com.br
}

\begin{abstract}
Resumo
Este artigo tem como proposta relacionar o papel da farmácia hospitalar diante de um cenário de pandemia, como a do novo coronavírus, e ressaltar a importância de um plano de contingência para o enfrentamento do impacto causado por esta pandemia em um hospital. A partir de uma revisão teórica, onde buscou-se trabalhos científicos e literaturas sobre o tema, em plataformas de pesquisa Scielo e Google Acadêmico, foi possível selecionar e analisar planos de contingência da farmácia hospitalar propostos em âmbito nacional e internacional, assim como artigos, livros, dados estatísticos, declarações, manuais, normas técnicas, dentre outros materias, que permeiam essa temática, correlacionando-os com as atribuições de um serviço de farmácia em unidades de saúde. Os resultados da revisão bibliográfica corroboram para as evidências da necessidade de se elaborar um plano de contingência específico para este cenário. Com isso concluiu-se que compete ao farmacêutico entender do processo assistencial e de gestão para se implementar e executar um plano de contingência na farmácia hospitalar específico para a pandemia por COVID-19. De forma a combater o vírus SARS-CoV-2 e mitigar seu impacto no âmbito hospitalar e na sociedade como um todo, garantindo a qualidade e a segurança dos pacientes e colaboradores.
\end{abstract}

Palavras-chave: Gestão em saúde; Serviço de farmácia hospitalar; Planos de contingência; Pandemia; COVID-19.

\begin{abstract}
This article aims to relate the role of hospital pharmacy in a pandemic scenario, such as the new coronavirus, and emphasize the importance of a contingency plan to face the impact caused by this pandemic in a hospital. From a theoretical review, where scientific works and literature on the subject were searched, in Scielo and Google Academic research platforms, it was possible to select and analyze contingency plans for hospital pharmacy proposed nationally and internationally, as well as articles, books, statistical data, statements, manuals, technical standards, among other materials that permeate this theme, correlating them with the attributions of a pharmacy service in health units. The results of the literature review corroborate the evidence of the need to develop a specific contingency plan for this scenario. Thus, it was concluded that it is up to the pharmacist to understand the care and management process to implement and execute a contingency plan in the hospital pharmacy specific for the COVID-19 pandemic. In order to fight the SARS-CoV-2 virus and mitigate its impact on the hospital environment and on society as a whole, ensuring the quality and safety of patients and employees.
\end{abstract}

Keywords: Health management; Hospital pharmacy service; Contingency plans; Pandemic; COVID-19.

\section{Resumen}

Este artículo tiene como objetivo relacionar el papel de la farmacia hospitalaria en un escenario pandémico, como el nuevo coronavirus, y enfatizar la importancia de un plan de contingencia para enfrentar el impacto que ocasiona esta pandemia en un hospital. A partir de una revisión teórica, donde se buscaron trabajos científicos y literatura sobre el tema, en las plataformas de investigación Scielo y Google Academic, fue posible seleccionar y analizar planes de contingencia para farmacia hospitalaria propuestos a nivel nacional e internacional, así como artículos, libros, datos estadísticos. , declaraciones, manuales, normas técnicas, entre otros materiales que impregnan esta temática, correlacionándolos con las atribuciones de un servicio de farmacia en las unidades de salud. Los resultados de la revisión 
de la literatura corroboran la evidencia de la necesidad de desarrollar un plan de contingencia específico para este escenario. Así, se concluyó que le corresponde al farmacéutico comprender el proceso de atención y gestión para implementar y ejecutar un plan de contingencia en la farmacia del hospital específico para la pandemia de COVID-19. Con el fin de combatir el virus SARS-CoV-2 y mitigar su impacto en el entorno hospitalario y en la sociedad en su conjunto, garantizando la calidad y seguridad de pacientes y empleados.

Palabras clave: Gestión de la salud; Servicio de farmacia hospitalaria; Planes de contingencia; Pandemia; COVID-19.

\section{Introdução}

Dentro da perspectiva de um possível colapso no sistema de saúde pública e privada, ocasionado pela rápida disseminação do vírus e seus graves efeitos, levando à hospitalização de milhares de pessoas em curto espaço de tempo, a farmácia hospitalar se encontra diante do desafio de desenvolver estratégias para garantir não só as atividades essenciais e prioritárias da assistência farmacêutica, como impedir a transmissão do vírus neste ambiente, sem colocar em risco os trabalhadores da saúde e demais prestadores de serviço em âmbito clínico-hospitalar.

Logo, fica evidente a grande importância de se estabelecer um plano de contingência na farmácia hospitalar e nos serviços de saúde, de forma que esse seja adaptável de acordo com a realidade local e aos diferentes contextos, situações e cenários que ainda possam vir a surgir durante a vigência dessa pandemia que acomete o mundo todo. Evitando não só o desabastecimento de serviços e insumos farmacêuticos, como flexibilizando o fluxo de atendimento e a rotina do setor, para a conter a difusão do vírus no local e a contaminação dos seus colaboradores. Garantindo então a qualidade da assistência farmacêutica e a saúde dos profissionais que atuam na linha de frente.

Tal esforço se justifica na realidade do cenário em que o mundo todo vive desde o final de 2019 quando foram registrados nos hospitais de Wuhan, cidade da província de Hubei na China, os primeiros casos de pneumonia de causa desconhecida que posteriormente teve seu agente etiológico isolado e identificado, o vírus SARS-CoV-2. Causador da COVID19 (Coronavirus Disease 2019), o novo coronavírus rapidamente se disseminou por toda Wuhan, epicentro da doença na China, e se espalhou pelo do mundo, chegando a registrar 178.202.610 casos confirmados de COVID-19 e 3.865.738 óbitos em todo o mundo até 21 de junho 2021 às 18 horas e 23 minutos, conforme dados publicados pela Organização Mundial de Saúde (Guedes, 2021).

Diante deste grande surto em 11 de março de 2020 a Organização Mundial da Saúde (OMS, 2020) classifica e declara essa doença infecciosa de alta consequência como uma pandemia, levando o mundo todo a uma grande busca pela prevenção, diagnósticos, tratamentos e cura dessa doença, de forma rápida e eficaz.

Após o primeiro caso de COVID-19 ser detectado em Wuhan em dezembro de 2019, passados aproximadamente um ano e sete meses, 174.502.686 casos de COVID-19 foram confirmados no mundo todo, incluindo 3.770.361 mortes, conforme relatos dos países à World Health Organization até 11 de junho de 2021 (WHO, 2021). Dentre esses, 17.296.118 casos de COVID-19 foram confirmados no Brasil e tendo ocorrido entre esses casos 484.235 mortes por COVID-19, segundo o Ministério da Saúde (Brasil, 2021) o que representa aproximadamente 9,9\% do total de casos do mundo, e corresponde a $8 \%$ da população brasileira que contraiu a doença, baseado na projeção de aproximadamente 213.197 .425 brasileiros de acordo com o Instituto Brasileiro de Geografia e Estatística (2021) em 11 de junho de 2021. (Brasil, 2021)

Entretanto, esses números podem estar fora da realidade em função dos desafios enfrentados pelas autoridades sanitárias na apuração dos casos, com isso, estima-se que o real número de mortos no mundo pelo novo coronavírus, seja "duas ou três vezes maior que os dados oficiais" de acordo com a diretora-geral da divisão de dados da OMS (Asma, 2021).

Enquanto não cessar essa pandemia, e diante dos dados apresentados, fica a preocupação com o colapso na saúde, mais especificamente na rede hospitalar, devido à falta de estrutura para o atendimento e tratamento adequado a pacientes com casos leves a graves, o que podem por consequência acarretar um desabastecimento de insumos farmacêuticos e uma ineficaz prestação dos serviços farmacêuticos. 
Este artigo tem como tema a importância de um plano de contingência na farmácia hospitalar frente à pandemia pelo SARS-CoV-2, síndrome respiratória aguda grave do coronavírus 2 (Severe Acute Respiratory Syndrome Coronavirus 2), para o enfrentamento do impacto que pode ser causado por essa pandemia em um hospital ou serviço de saúde, relacionando o papel da farmácia hospitalar com a necessidade de manter e dar continuidade na prestação de serviços farmacêuticos diante deste cenário, bem como minimizar riscos e prevenir a ocorrência de doenças e agravos em pacientes e prestadores de serviço.

Sendo assim, este trabalho visa relacionar o papel da farmácia hospitalar diante da pandemia da COVID-19, ressaltando a importância de se implantar um plano de contingência específico para o enfrentamento dos possíveis impactos causados por essa pandemia, através de uma revisão teórica acerca de planos de contingência propostos em âmbito nacional e internacional, assim como demais referências bibliográficas acerca desta temática.

\section{Metodologia}

A construção deste artigo se desenvolveu a partir do referencial teórico, utilizando como instrumento metodológico a pesquisa bibliográfica que segundo Gil (2010, p.29-31) “[...] é elaborada com base em material já publicado. Tradicionalmente, esta modalidade de pesquisa inclui material impresso como livros, revistas, jornais, teses, dissertações e anais de eventos científicos".

Sendo assim, buscou-se através das plataformas de pesquisa SCIELO e GOOGLE ACADÊMICO materiais dos anos 2000 até a atualidade, divulgados através do meio eletrônico, em língua portuguesa e inglesa. Foram então selecionados materiais como manuais e normas técnicas, procedimentos operacionais, legislações, dados estatísticos, declarações de instituições de saúde, entrevistas, artigos científicos, teses e livros que abordam especificamente sobre o estabelecimento de planos de contingência adotados em farmácia hospitalar para combate ao impacto da covid-19 no âmbito hospitalar, assim como os que falam sobre a gestão em farmácia hospitalar, assistência farmacêutica, ciclo de assistência farmacêutica, pandemia pela covid19 e impactos causados pela covid-19 nos hospitais.

O material resultante desta pesquisa bibliográfica foi minuciosamente analisado e selecionado para a construção deste artigo, sendo baseado nas ideias e concepções de autores como Santos (2012), Andrade (2015), Mendonça (2020), Brasil (2010), OMS (2020), Conselho Federal de Farmácia (2020, 2021), Ordem dos Farmacêuticos (2020), Sociedade Brasileira de Farmácia Hospitalar $(2007,2020)$, dentre outros, disponibilizando uma publicação com a proposta de apoiar a farmácia hospitalar, assim como farmacêuticos, gestores e colaboradores para adotarem medidas assertivas diante do enfrentamento da COVID-19, de forma a "garantir a segurança dos pacientes, dos trabalhadores da saúde e da população, e a prestação de serviços, mantendo o acesso a medicamentos, produtos para a saúde e cuidado em saúde”, segundo Conselho Federal de Farmácia (2020).

\section{Resultados e Discussão}

A farmácia hospitalar é caracterizada, sob o viés da gestão, como uma unidade técnico-administrativa de uma organização clínico-hospitalar, onde processam atividades relacionadas com compras, armazenamento, economia, produção, controle, custos, dentre outras. Entretanto sob a ótica do cuidado à saúde, a farmácia hospitalar também é uma unidade clínicoassistencial responsável por promover a saúde, o uso racional de medicamentos, a orientação aos pacientes, o acesso a insumos e medicamentos, dentre outras. Compreendendo, essas duas faces da farmácia hospitalar, no processo de assistência farmacêutica, que tem seu sucesso exatamente no equilíbrio entre a gestão e o cuidado à saúde. (Andrade, 2015; Brasil, 2010)

De acordo com a portaria $n^{\circ} 4.283$, publicada em 2010 pelo Ministério da Saúde, que aprova as diretrizes e estratégias para organização, fortalecimento e aprimoramento das ações e serviços de farmácia no âmbito dos hospitais:

Farmácia hospitalar: é a unidade clínico-assistencial, técnica e administrativa, onde se processam as atividades 
relacionadas à assistência farmacêutica, dirigida exclusivamente por farmacêutico, compondo a estrutura organizacional do hospital e integrada funcionalmente com as demais unidades administrativas e de assistência ao paciente (Brasil, 2010).

Mais especificamente, segundo Andrade (2015) as etapas do Ciclo de Assistência Farmacêutica ocorrem no setor da farmácia hospitalar, local onde:

[...] se processam atividades relacionadas à Assistência Farmacêutica, à produção, ao armazenamento, ao controle, à dispensação, à distribuição de medicamentos e correlatos às unidades hospitalares; bem como à orientação de pacientes internos e ambulatoriais visando sempre a eficácia da terapêutica, além da redução dos custos, voltando-se, também, para o ensino e a pesquisa, propiciando um vasto campo de aprimoramento profissional.

Considerando as tecnologias da saúde como um "Conjunto de equipamentos, de medicamentos, de insumos e de procedimentos, utilizados na prestação de serviços de saúde" (Brasil, 2010), como fatores essenciais na prestação desses serviços, pode-se afirmar que a farmácia hospitalar é um setor vital no âmbito clínico-hospitalar. Desta Forma, baseado na abrangência de seus serviços e responsabilidades, a gestão de uma farmácia hospitalar é algo de grande importância, visto que neste setor é feita a seleção, programação, aquisição, armazenamento, distribuição, prescrição, manipulação e a dispensação dos insumos mais caros de um hospital: os medicamentos, correlatos e materiais médico-hospitalares (Santos, 2012).

Neste cenário, para que se tenha um bom funcionamento e prestação de serviços de saúde, bem como a garantia do cuidado à saúde e da segurança do paciente, a sua gestão e planejamento de uma farmácia hospitalar devem ser pautadas no principal objetivo de:

[...] contribuir no processo de cuidado à saúde, melhorando a qualidade da assistência prestada ao paciente, promovendo o uso seguro e racional de medicamentos e produtos para a Saúde; também é responsável pelo armazenamento, distribuição, dispensação e controle de todos os medicamentos e produtos para saúde usados pelos pacientes internados e ambulatoriais do hospital, bem como, pelo fracionamento e preparo de medicamentos. (Sociedade Brasileira de Farmácia Hospitalar [SBRAFH], 2007)

Com a pandemia do novo coronavírus, de acordo com a Organização Mundial da Saúde (OMS, 2020) 80\% dos infectados podem ser assintomáticos, e somente $20 \%$ dos casos, devido a dificuldades respiratórias, podem necessitar de atendimento hospitalar, podendo 5\% desses apresentar insuficiência respiratória necessitando de suporte ventilatório. O rápido aumento no número de casos e na procura por atendimento hospitalar para tratamentos de casos leves a graves de COVID-19 tem provocado o aumento na demanda do serviço de saúde e a superlotação dos leitos hospitalares. E com isso a farmácia hospitalar se vê diante do desafio, nunca visto antes, de enfrentar os impactos causados pelo COVID-19 nos hospitais, o que vai exigir um novo planejamento de gestão do setor para que possa cumprir sua missão enquanto unidade técnico-administrativa e clínico-assistencial.

Diante deste cenário, a demanda na necessidade de medicamentos, insumos farmacêuticos e materiais necessários para o atendimento e tratamento dos pacientes, aumentou exponencialmente. Assim como a necessidade de todos os Equipamentos de Proteção Individuais - EPIs de funcionários e pacientes, os quais são administrados pela farmácia hospitalar, para se evitar o contágio e transmissibilidade do vírus no âmbito hospitalar, entre pacientes, profissionais da saúde e colaboradores. Por exemplo, várias denúncias sobre falta de EPIs puderam ser apuradas de acordo com o levantamento de dados junto a Associação Médica Brasileira realizado pelo Portal G1 (2020):

Um levantamento obtido pelo G1 aponta que $87 \%$ das denúncias de médicos sobre falta de equipamentos de proteção individual (EPI) são de máscaras N95, um tipo de material indicado a profissionais de saúde no atendimento à pacientes com o novo coronavírus. Em seguida, vêm óculos do tipo face shield (70\%) e capote impermeável (66\%). Os dados são da Associação Médica Brasileira (AMB) e se referem ao período de 19 de março a 12 de abril. 
Segundo a Sociedade Brasileira de Farmácia Hospitalar e Serviços de Saúde - SBRAFH, muitas dificuldades surgiram em decorrência da pandemia pela COVID-19, e com isso todos os processos essenciais à assistência no sistema de saúde hospitalar necessitaram ser reexaminar e readequados. (Mendonça, 2020).

Dentre esses processos, vale destacar a readequação de fluxos e rotinas dos serviços, como a dispensação de medicamentos e insumos hospitalares, garantindo que cheguem aos pacientes, sem que haja o risco de contaminação. Assim como garantindo que a devolução dos medicamentos excedentes retorne à farmácia desinfectados, obedecendo aos critérios de segurança e prezando pela segurança dos profissionais direta e indiretamente envolvidos neste processo. "Como alternativa, os produtos poderão ser colocados em quarentena, a qual deverá respeitar o período em que o vírus permanece viável frente aos diversos tipos de superfície" (Kampf, et al., 2020; Van Doremalen, et al., 2020; WHO, 2020; Costa, 2020).

Outro ponto crítico que já ocorre nos hospitais de todo o país são as evidências no desabastecimento de medicamentos utilizadas no manejo dos pacientes internados com COVID-19 e outras doenças, bem como de medicamentos utilizados no cuidado em Unidades de Terapia Intensiva, principalmente para os processos de intubação de pacientes com complicações decorrentes do novo coronavírus, como sedativos, analgésicos e bloqueadores neuromusculares. Além da falta de oxigênio medicinal, que é extremamente necessário para um dos pilares de tratamento da COVID-19, a oxigenioterapia, segundo a Associação de Medicina Intensiva Brasileira (2021).

"Para além da iminente crise de oxigênio generalizada em todo o país, o Conselho Federal de Farmácia (CFF) evidencia a necessidade de reabastecimento de medicamentos usados em UTIs, tanto para pacientes internados pela covid-19 quanto por outras doenças" (CFF, 2021).

A Confederação Nacional de Saúde (2020) diz que "temos recebido informações de grandes redes que têm tido dificuldade de comprar esse tipo de medicamento", relatando a falta de dezessete tipos de sedativos. "Os hospitais precisam desse medicamento porque não conseguem fazer a intubação. Faz-se um esforço enorme para criar leitos, comprar respiradores, e aí não consegue entubar?” indaga a Confederação Nacional de Saúde (2020) em entrevista ao Portal Setor Saúde.

Diante desses relatos e de acordo com o Diretor de Comunicação da SBRAFH, a farmácia hospitalar necessita de esforços adicionais:

Esse cenário de adversidades tem exigido do serviço de Farmácia Hospitalar esforços adicionais quanto à necessidade de disponibilização de alternativas terapêuticas na tentativa de suprir tal necessidade ao mesmo tempo que fortaleceu o trabalho em equipe nas interfaces entre os serviços assistenciais e da gestão de suprimentos (Mendonça, 2020).

Nesses tempos difíceis, diante de um cenário não habitual para a farmácia hospitalar, esta que se encontra na linha de frente do combate ao novo coronavírus passa a ter novas responsabilidade no processo de Assistência Farmacêutica, que:

[...] permeiam desde aspectos regulatórios quanto a controle sanitário e legal do estabelecimento de saúde, controle de qualidade e validação de produtos e processos, passando pela gestão de demandas, de estoques, dispensação de medicamentos e insumos farmacêuticos, participação em comissões multidisciplinares, chegando até o acompanhamento clínico do paciente, avaliando e padronizando, junto à equipe multiprofissional, terapias efetivas e seguras, embasadas por protocolos clínicos, fundamentados por evidências científicas, a fim de primar pela segurança e qualidade de vida dos pacientes (Mendonça, 2020).

O que corrobora a importância de se implantar um plano de contingência na farmácia hospitalar para o enfrentamento dos impactos causados pela pandemia do Covid-19 na rede hospitalar pública e privada, e demais unidades de saúde. E, mais uma vez, de acordo com a portaria ${ }^{\circ}$ 4.283, publicada em 2010 pelo Ministério da Saúde, o conceito de Plano de contingência consiste em um:

[...] plano que descreve as medidas a serem tomadas, em momento de risco, por um estabelecimento de saúde, incluindo 
a ativação de processos manuais, para fazer em que os processos vitais voltem a funcionar plenamente, ou num estado minimamente aceitável, o mais rapidamente possível, evitando paralisação prolongada que possa gerar danos aos pacientes ou prejuízos financeiros à instituição (Brasil, 2010).

Os planos de contingência são feitos para serem implementados em situações de emergência como a de uma pandemia por exemplo, ao qual o mundo está vivendo na atualidade deste trabalho. E o seu objetivo principal é traçar um planejamento de ações coordenadas para serem tomadas conforme os níveis de resposta (SBRAFH, 2020).

Esse plano serve como um instrumento de gestão para organizar, uniformizar, facilitar, agilizar e orientar essas ações coordenadas que são necessárias para combater o impacto causado pela pandemia da COVID-19, de forma a adotar medidas de contenção para impedir ou minimizar os riscos do funcionamento do serviço de farmácia em um hospital e da segurança dos pacientes e colaboradores.

Torna-se necessário, então, "Identificar e prover medidas de segurança para os profissionais designados para a realização das tarefas na implementação do Plano; estabelecer protocolos e procedimentos operacionais necessários para a realização das ações frente ao período de calamidade pública" (Universidade Federal de Minas Gerais, 2020).

Sendo assim, embora o plano de contingência de uma farmácia hospitalar deva ser adaptado de acordo com a realidade local, de cada instituição e da situação que estiver vivenciando, pode-se concluir que é preciso alcançar os objetivos específicos para um plano de contingência como este, que são:

Fortalecer a área de apoio para manutenção das atividades farmacêuticas a fim de auxiliar as equipes da linha de frente; Planejar a gestão dos recursos humanos neste cenário, prevendo rotação da equipe, trabalhos extras, atividades remotas ou ausências, definindo também quais serão os serviços essenciais e prioritários; Assegurar a dispensação de medicamentos e produtos para saúde, considerando a possibilidade de escassez de recursos; Manter constante contato com as equipes assistenciais e demais equipes de apoio, a fim de dispor itens substitutos no cenário de escassez; Considerar a assistência farmacêutica nos hospitais de campanha; Apresentar a assistência farmacêutica no âmbito hospitalar veterinário dentro do contexto da pandemia do novo coronavírus (SBRAFH, 2020).

E de acordo com o preconizado pelo Conselho do Colégio de Especialidade de Farmácia Hospitalar da Ordem dos Farmacêuticos de Portugal, esses objetivos e finalidades são:

a. Preparar a resposta das Farmácias Hospitalares para a epidemia de COVID-19 em Portugal; b. Reduzir a disseminação da infeção, através da promoção de medidas de saúde pública, individuais ou comunitárias; c. Assegurar a terapêutica medicamentosa a um elevado número de doentes com critérios de qualidade, eficácia e segurança; d. Trabalhar, em permanência, em equipas transdisciplinares, adaptando o funcionamento e resposta das farmácias hospitalares às necessidades da instituição e à evolução da situação; e. Antecipar impactos na rotina diária da farmácia, ensaiando protocolos de atuação de acordo com diferentes estádios de evolução da epidemia; f. Estas orientações devem ser adaptadas à realidade de cada instituição e ao evoluir natural das situações, não devendo ser de alguma forma interpretadas como uma norma rígida ou de aplicação direta (Ordem dos Farmacêuticos, 2020).

Então, baseado em planos de contingência propostos no Brasil e em Portugal pela Sociedade Brasileira de Farmácia Hospitalar (2020) e Ordem dos Farmacêuticos (2020), respectivamente, este contingenciamento deve estar pautado nas premissas que abarcam as atividades farmacêuticas de gestão e recursos humanos; higienização de mão e desinfecção de superfícies; uso de equipamentos de proteção individual; formação e informação transversal a toda a equipe; ciclo logístico do medicamento e produtos para a saúde: seleção, programação, aquisição, recepção e armazenamento, manipulação, prescrição, distribuição, dispensação e devoluções; circuito de medicamento experimental; fluxo da distribuição, dispensação e armazenamento de estupefacientes, psicotrópicos, benzodiazepínicos, hemoderivados; farmácia clínica; assistência farmacêutica hospitalar e ambulatorial; assistência farmacêutica em hospitais de campanha, assistência farmacêutica em hospital veterinário.

Tais circunstâncias revelam que a criação de um plano de contingência surge como um instrumento de combate a COVID-19 motivado pelo maior desafio já enfrentado pela farmácia hospitalar na atualidade, que tem sido o de "promover 
estratégias como forma de interromper o ciclo de transmissão do vírus, bem como impulsionar o fluxo na promoção da saúde em pacientes com COVID-19 e, com isso, reduzir a sobrecarga das unidades de urgência e emergência" (SBRAFH, 2020). De forma a não colocar seus colaboradores e pacientes em risco e tampouco reduzir a qualidade do serviço ofertado.

Sem dúvida nenhuma, as relações e processos indispensáveis à gestão desta área precisaram estar ainda mais integrados aos demais serviços de apoio, bem como assistenciais. O "pensar" em assistência farmacêutica necessita de padrões de processos, fluxos, protocolos para uso efetivo e seguro de medicamentos e produtos para a saúde, sempre considerando a interface com as demais áreas hospitalares e o acesso racional e seguro a estas tecnologias em saúde (Mendonça, 2020).

\section{Conclusão}

Uma visão regulatória técnico-administrativa, científica, clínico-assistencial e para o controle sanitário se faz necessária frente a gestão de uma farmácia hospitalar no âmbito da pandemia por COVID-19. Compete ao farmacêutico, então, o conhecimento assistencial e de gestão, para atingir a motivação maior do seu trabalho na área hospitalar, que é o de garantir aos pacientes assistidos pela respectiva unidade de saúde, as melhores resoluções clínicas possíveis, com qualidade e segurança a esses e aos demais envolvidos.

Conclui-se então, que a farmácia hospitalar, parte integrante do ciclo de assistência hospitalar, depende diretamente da organização e do sincronismo no próprio setor e entre os demais serviços e profissionais inseridos na rede hospitalar, ainda mais em situações como a da pandemia por COVID-19. E para isso a elaboração de um plano de contingência específico se torna indispensável, o que é corroborado pelos evidentes fatos apresentados nesta pesquisa.

Logo a implementação e execução de um Plano de Contingência específico para a pandemia por COVID-19 na farmácia hospitalar, torna-se, associado a outras ações, uma importante ferramenta para o combate ao vírus SARS-CoV-2 e para a mitigação do seu impacto no âmbito hospitalar e na sociedade como um todo.

Como sugestão para futuros trabalhos, a partir deste artigo, é possível desenvolver e aplicar um plano de contingência nas Farmácias Hospitalares, assim como desenvolver estudos mais profundos analisando a aplicabilidade desse plano de contingência, conforme proposto, sua eficiência e resultados, bem como traçar um comparativo entre farmácias hospitalares que adotaram ou não o plano de contingência e seus respectivos impactos diante do cenário de pandemia pelo COVID-19 ao qual o mundo ainda está vivendo até o momento da publicação deste artigo, contribuindo então não só para a classe científica, mas para a população, garantindo a essa segurança, qualidade de vida e saúde.

\section{Referências}

Andrade, L. B. (2015). O papel do farmacêutico no âmbito hospitalar. https://www.ccecursos.com.br/img/ resumos/o-papel-do-farmac-utico-no--mbitohospitalar. Pdf

Asma, S. (2021). OMS: número de mortes por covid no mundo é até 3 vezes maior que dados oficiais. https://epocanegocios.globo.com/Mundo/noticia/ 2021/05/epoca-negocios-oms-numero-de-mortes-por-covid-no-mundo-e-ate-3-vezes-maior-que-dados-oficiais.html

Associação de Medicina Intensiva Brasileira. (2021). Orientações sobre o uso racional do gás oxigênio em pacientes graves com suspeita de infecção por SARSCoV-2. https://www.amib.org.br/fileadmin/user_upload/amib/2021/janeiro/27/orientacoes_sobre_o_uso_racional_do_gas_oxigenio_em_pacientes_grav es_com_suspeita_de_infeccao_por_sars-cov-2vjs.pdf

Brasil. Ministério da Saúde. (2021). Painel Coronavírus. https://covid.saude.gov.br/

Brasil. Ministério da Saúde. (2010). Portaria $n^{o}$ 4.283, de 30 de dezembro de 2010. Diário Oficial da União, Brasília, 31 dez. 2010. http://bvsms. saude.gov.br/bvs/saudelegis/gm/2010/prt4283_30_12_2010.html

Confederação Nacional de Saúde. (2020). Desabastecimento de medicamentos essenciais no combate à covid-19 preocupa hospitais. https://setorsaude. com.br/desabastecimento-de-medicamentos-essenciais-no-combate-a-covid-19-preocupa-hospitais/

Conselho Federal de Farmácia. (2020). Atuação do farmacêutico frente à pandemia da Doença Causada pelo Coronavírus Plano de resposta para a farmácias privadas e públicas da Atenção Primária. https://www.cff.org.br/userfiles/Coronav\%C3\%ADrus\%20orienta\%C3\%A7\%C3\% B5es\%20a\%20 Farm\%C3\% A1cias $\% 20 \mathrm{da} \%$ 20APS $\% 20$ no\%20SUS\%20(1).pdf 
Research, Society and Development, v. 10, n. 7, e59610717098, 2021

(CC BY 4.0) | ISSN 2525-3409 | DOI: http://dx.doi.org/10.33448/rsd-v10i7.17098

Conselho Federal de Farmácia. (2021). Conselho Federal de Farmácia alerta para falta de medicamentos usados nas UTIs. https://www.correio braziliense.com.br/brasil/2021/03/4912672-conselho-federal-de-farmacia-alerta-para-falta-de-medicamentos-usados-nas-utis. Html

Costa. T. (2020). Devolução de medicamentos de áreas destinadas ao tratamento de covid-19. https://www.gov.br/ebserh/pt-br/hospitais-universitarios/regiaocentro-oeste/hc-ufg/governanca/pops-e-protocolos/gerencia-de-atencao-a-saude/26POP.UFCDIS.022DevoluodemedicamentosCovid19atual.pdf

Gil, Antonio Carlos. (2010). Como elaborar projetos de pesquisa. (5a ed.), Atlas, 2010. 184p

Guedes, B. M., da Silva, T. B., \& Dal Sasso, M. A. (2021). Casos suspeitos e confirmados do novo coronavírus (covid-19) registrados no mundo, no Brasil e na rede Ebserh. Vigilância em Foco. https://www.gov.br/ebserh/pt-br/saude/covid-19/VigilnciaemFocoedioCovid19n246de21062021.pdf.

Instituto Brasileiro de Geografia e Estatística (2021). População do Brasil. https://www.ibge.gov.br/apps/populacao/projecao/box_popclock.php

Kampf, G. et al. (2020). Persistence of coronaviruses on inanimate surfaces and its inactivation with biocidal agents. Journal of Hospital Infection, v. 104, pag. 246-25. https://www.journalofhospitalinfection.com/article/S0195-6701(20)30046-3/pdf

Mendonça, L. F. R. de. (2020). Os desafios da farmácia hospitalar com a covid-19. https://www.anbfarma.com. br/noticia/os-desafios-da-farmacia-hospitalarcom-a-covid-19

OMS, O. (2020). Manual de orientações da covid-19 (vírus SARS-CoV-2). https://www.saude.sc.gov.br/coronavirus/arquivos/Manual_23-10-atualizado.pdf

OMS, O. (2020). OMS afirma que COVID-19 é agora caracterizada como pandemia. https://www.paho.org/bra/index.php? option=com_content\&view= article \&id=6120:oms-afirma-que-covid-19-e-agora-caracterizada-comopandemia \& Itemid=812

Ordem dos Farmacêuticos. (2020). Plano de contingência da Farmácia Hospitalar no âmbito da pandemia COVID-19. https://www.ordemfarmaceuticos. pt/fotos /editor2/covid_ 19/planos/PlanoFH.pdf

Portal G1 (2020). 87\% das denúncias de falta de equipamentos de proteção são sobre máscaras N95, diz associação. https://g1.globo.com/bemestar/ coronavirus/noticia/2020/04/15/87percent-das-denuncias-de-falta-de-equipamentos-de-protecao-sao-sobre-mascaras-n95-diz-associacao.ghtml

Santos, G. A. A. (2012). Gestão de farmácia hospitalar. Senac, 2012

Sociedade Brasileira de Farmácia Hospitalar. (2007). Padrões mínimos para farmácia hospitalar. http://www.sbrafh.org.br/site/public/temp/4f7baaa6b 63d5.pdf

Sociedade Brasileira de Farmácia Hospitalar. (2020). Plano de contingência em diversos cenários farmacêuticos no âmbito da pandemia por COVID-19. http://www.sbrafh. org.br/inicial/ plano-de-contingencia-em-diversos-cenarios farmaceuticos-no-ambito-da-pandemia-por-covid-19/

Universidade Federal de Minas Gerais. (2020). Plano de contingência da farmácia do hospital veterinário da UFMG no âmbito da pandemia por covid-19. https://vet.ufmg.br/ARQUIVOS/FCK/file/Plano\%20de\%20 Conting\%c3\%aancia\%20Farm\%c3\%a1 cia\%20Hospital\%20Veterin\%c3\%a1rio\%20UFMG.pdf

Van Doremalen, N., et al. (2020). Aerosol and surface stability of SARS-CoV-2 as compared with SARS-CoV-1. The New England Journal of Medicine. https://portaldeboaspraticas.iff.fiocruz.br/wp-content/uploads/2020/06/nejmc2004973.pdf

WHO, W. (2021). Coronavirus (COVID-19) Dashboard. https://covid19.who.int/

WHO, W. (2020). Rational use of personal protective equipment for coronavirus disease (COVID-19) and considerations during severe shortages: interim guidance, 6 April 2020. https://apps.who.int/iris/handle/10665/331695 